\title{
Does Low-Level Laser Therapy Decrease Muscle-Damaging Mediators After Performance in Soccer Athletes Versus Sham Laser Treatment? A Critically Appraised Topic
}

\author{
Jordan Bettleyon and Thomas W. Kaminski
}

\begin{abstract}
Clinical Scenario: Low-level laser therapy (LLLT) is a controversial topic for its use in athletic recovery, mainly due to inconsistency in research regarding the application of LLLT. Articles on LLLT have assessed its effectiveness in untrained humans through pain scales, functional scales, and blood draws, and it has been found capable in nonathletic rehabilitative use. The controversy lies with LLLT in the recovering athlete. Not only do athletes need to perform at high levels, but each sport is unique in the metabolic demands placed on the athletes' bodies. This modality can alter chemical mediators of the inflammatory process, specifically blood lactate (BL) and creatine kinase (CK). During soccer contests, it is a common problem for athletes to have an average $\mathrm{CK}$ level of $800 \mathrm{U} / \mathrm{L}$ and $\mathrm{BL}$ of $8 \mathrm{mmol} \cdot \mathrm{L}$, increasing delayed-onset muscle soreness and fatigue. Micro-CK level elevation is associated with cellular membrane damage, localized hypoxia, and electrolyte imbalances, hindering the recovery process. Clinical Question: Does LLLT decrease muscle-damaging mediators effecting player fatigue and delayed-onset muscle soreness after performance in soccer athletes versus sham treatment? Summary of Key Findings: In 3 studies, preperformance, postperformance, or preperformance and postperformance LLLT was performed and evaluated BL ( 2 of 3 ) and CK ( 2 of 3 ). In each article, BL and CK showed a significant decrease $(P<.05)$ when performed either preperformance or postperformance versus the control group. The greatest decrease in these mediators was noticed when postperformance laser therapy was performed. Clinical Bottom Line: LLLT at 10, 30, or $50 \mathrm{~J}$ performed at a minimum of 2 locations on the rectus femoris, vastus lateralis, and vastus medialis bilaterally for 10 seconds each is significant in decreasing blood serum levels of BL and CK when performed postexercise. Strength of Recommendations: All 3 articles obtained a Physiotherapy Evidence Database score of $\geq 8 / 10$.
\end{abstract}

Keywords: recovery, creatine kinase, blood lactate

\section{Clinical Scenario}

Low-level laser therapy (LLLT) has been a controversial topic for its use in athletic recovery, mainly due to the inconsistency in research regarding the timing of application for this modality. Articles on LLLT have assessed its effectiveness in untrained humans through pain scales, functional scales, and blood draws, and it has been found capable in nonathletic rehabilitative use. The controversy lies with LLLT use in the recovering athlete, who needs to perform at peak levels on a day-to-day basis. Not only do athletes need to perform at high levels, but each sport is unique in the metabolic demands placed on the athletes' bodies; this is where the timing of LLLT is controversial. Timing of this modality can alter chemical mediators of the inflammatory process and inhibit damaging enzymes, more specifically, blood lactate (BL) and creatine kinase (CK). During soccer contests, it is a common problem for field athletes to have an average CK level of $800 \mathrm{U} / \mathrm{L}$ and $\mathrm{BL}$ of $8 \mathrm{mmol} \cdot \mathrm{L}$, increasing delayed-onset muscle soreness (DOMS) and muscular fatigue. ${ }^{1,2}$ Micro-CK level elevation is also associated with cellular membrane damage, localized hypoxia, and disruption of electrolyte balances, causing a stunting of the recovery process. ${ }^{3}$ For these reasons, $\mathrm{CK}$ and $\mathrm{BL}$ are precipitators of muscle fatigue and function and

Bettleyon is with the Department of Athletics, Methodist University, Fayetteville, NC, USA. Kaminski is with KAAP, University of Delaware, Newark, DE, USA. Bettleyon (jbettleyon@methodist.edu) is corresponding author. were selected for the specified purpose of controlling these mediators that cause DOMS, fatigue, and delayed recovery responses with LLLT.

\section{Focused Clinical Question}

Does LLLT decrease muscle-damaging mediators effecting player fatigue and DOMS after performance in soccer athletes versus sham treatment?

\section{Clinical Bottom Line}

The LLLT either at 10,30 , or $50 \mathrm{~J}$ performed at a minimum of 2 locations on the rectus femoris, vastus lateralis, and vastus medialis bilaterally for 10 seconds each is significant in decreasing blood serum levels of BL and CK when performed postexercise. ${ }^{4-6}$

\section{Search Strategy}

\section{Guided Search terms}

- Patient/Client group: male or female soccer athletes, collegiate soccer athletes, professional college athletes

- Intervention: low-level laser therapy, light therapy

- Comparison: sham, no low-level laser therapy

- Outcome: creatine kinase, blood lactate 


\section{Databases Searched}

-PubMed

-Google Scholar

-ScienceDirect

-Physiotherapy Evidence Database (PEDro)

\section{Inclusion and Exclusion Criteria}

\section{Inclusion Criteria}

- Use of LLLT

- Evaluation of $\mathrm{CK}$ or BL

- LLLT on soccer athletes

- Evaluation of chemical mediators after simulated or actual performance

- PEDro score $\geq 5$

\section{Exclusion Criteria}

- Animal studies

- Use of another modality in conjunction with LLLT

\section{Search Results}

After searching the included databases, 3 studies were identified with PEDro scores of $\geq 8$ and included in this appraisal. Table 1 shows the individual breakdown of scores.

\section{Summary of Best Evidence}

The LLLT was effective in decreasing serum CK and BL when applied preperformance or postperformance; however, the greatest decrease was evident when laser therapy was performed postperformance (Table 2). ${ }^{4-6}$

\section{Implications for Practice, Education, and Future Research}

Blood lactate is a byproduct of anaerobic glycolysis and has been blamed for DOMS; however, BL is normally filtered and converted back into pyruvate within 6- to 24-hours postexercise in trained individuals. ${ }^{7}$ The enzyme responsible for this conversion is known as lactate dehydrogenase (LDH). LDH is found in liver, heart, brain, and muscle cell cytoplasm and changes the molecular structure of BL $\left(\mathrm{C}_{3} \mathrm{H}_{6} \mathrm{O}_{3}\right)$ to pyruvate $\left(\mathrm{C}_{3} \mathrm{H}_{4} \mathrm{O}_{3}\right) .{ }^{8}$ When conversion of $\mathrm{BL}$ takes place in muscular tissue, $\mathrm{LDH}$ is primed by nicotinamide adenine dinucleotide (NAD+), which is a metabolic transporter in energy production within the mitochondria. ${ }^{7,8}$ Laser therapy is able to stimulate the mitochondrial respiratory chain, which signals NAD+ to the mitochondria. ${ }^{9}$ With increased signaling of the NAD+ primer, $\mathrm{LDH}$ is able to convert $\mathrm{BL}$ into pyruvate regardless if preperformance or postperformance laser therapy is performed on athletes, specifically in the quadriceps muscles of soccer players. Dos Reis et $\mathrm{al}^{4}$ recorded a significant difference in BL when LLLT is performed postperformance
Table 1 Articles Score

\begin{tabular}{llc}
\hline Article & Study design & PEDro score \\
\hline Dos Reis et al ${ }^{4}$ & $\begin{array}{l}\text { Randomized, double-blind, } \\
\text { placebo-controlled trial }\end{array}$ & $8 / 10$ \\
Leal-Junior et al & $\begin{array}{l}\text { Randomized, double-blind, } \\
\text { placebo-controlled, crossover } \\
\text { trial }\end{array}$ & $8 / 10$ \\
Aver Vanin et al & $\begin{array}{l}\text { Randomized, double-blind, } \\
\text { placebo-controlled trial }\end{array}$ & $10 / 10$ \\
\hline
\end{tabular}

Abbreviation: PEDro, Physiotherapy Evidence Database.

when compared with placebo $(P<.01)$. Even though BL was lower versus placebo when LLLT was performed preexercise, there was no clinically significant difference. BL levels after performance in dos Reis et $\mathrm{al}^{4}$ were placebo-4 $\mathrm{mmol} / \mathrm{L}$ after 10 minutes, prelaser group $-3 \mathrm{mmol} / \mathrm{L}$ after 10 minutes, and postlaser group $-2 \mathrm{mmol} / \mathrm{L}$ after 10 minutes. Leal-Junior et $\mathrm{al}^{5}$ noticed BL levels decreased after 15 minutes when LLLT was performed pre-Wingate test when compared with placebo $(P<$ $.01)$ : placebo-10.52 mmol.L and laser group-8.55 mmol.L. The greatest difference in BL levels came when LLLT was performed postexercise.

Creatine kinase is an enzyme that can be considered either good or bad to the body. CK is associated with muscle damage after strenuous exercise and has been linked to DOMS. ${ }^{3} \mathrm{CK}$ is also used to convert adenosine diphosphate to adenosine triphosphate (ATP) using creatine phosphate. , $^{3,8}$ This conversion is the first mechanism for producing energy when the body begins exercise. When the body is at rest, a CK polymer (mitochondrial creatine kinase) is used to reconvert ATP and creatine back to adenosine diphosphate and creatine phosphate for future energy use. ${ }^{3}$ To create adenosine diphosphate and creatine phosphate for future use, there needs to be an abundance of ATP readily accessible. By performing laser therapy over the muscle group that was worked, cytochrome $\mathrm{c}$ and the rest of the mitochondria are stimulated to continue making ATP for energy use. ${ }^{10,11}$ By producing ATP, CK is utilized to store energy, creating molecules for future energy use. Aver Vanin et $\mathrm{al}^{6}$ noticed significant decreases $(P<.05)$ in $\mathrm{CK}$ levels when laser therapy was performed at 10 and $50 \mathrm{~J}$ preexercise at 24-, 48-, 72-, and 96-hour time intervals. Dos Reis et $\mathrm{al}^{4}$ recorded $\mathrm{CK}$ levels that decreased significantly when laser therapy was performed postexercise compared with placebo within 15 minutes on day 8 (second session): placebo-400 U/L, prelaser-200 U/L, and postlaser-100 U/L. The greatest effects of laser therapy in reducing $\mathrm{CK}$ levels and utilizing $\mathrm{CK}$ were observed when postexercise LLLT was performed.

Performing either preperformance or postperformance laser therapy can decrease CK, and postexercise LLLT decreases BL. The greatest effects from laser therapy are performing postperformance therapy at 10,30 , or $50 \mathrm{~J}$ of total energy performed at a minimum of 2 locations on the rectus femoris, vastus lateralis, and vastus medialis bilaterally for 10 seconds each. Further research is needed to assess the reliability and validity of the use of LLLT in rehabilitation and recovery in other sports. Further research is also needed to assess BL and CK when postexercise LLLT is performed and to evaluate the differences in male versus female soccer players. 
Table 2 Study Parameters and Results

\begin{tabular}{ll}
\hline & Dos Reis et al $^{4}$ \\
\hline Participants & 27 male soccer athletes \\
Exercise & $75 \% 1-\mathrm{RM}$ knee extension to fatigue \\
LLLT frequency & $830 \mathrm{~nm}$ \\
LLLT dosage & -Placebo: 0 J per leg each session $(0 \mathrm{~J}$ at \\
& each site) $10 \mathrm{~s}$ at each of 42 points on \\
& quadriceps on days 1 and 8 \\
& -Preexercise laser group: $25.2 \mathrm{~J}$ per leg \\
& each session $(0.6 \mathrm{~J}$ at each site) $10 \mathrm{~s}$ at \\
& each of 42 points on quadriceps on days \\
& 1 and 8 \\
& - Postexercise laser group: $25.2 \mathrm{~J}$ per leg \\
& each session $(0.6 \mathrm{~J}$ at each site) $10 \mathrm{~s}$ at \\
& each of 42 points on quadriceps on days \\
& 1 and 8
\end{tabular}

Leal-Junior et al $^{5}$

11 male soccer athletes

30 s maximal Wingate

$830 \mathrm{~nm}$

-Placebo: $0 \mathrm{~J}$ per leg ( $0 \mathrm{~J}$ at each site) $30 \mathrm{~s}$ at each of the 5 points on bilateral rectus femoris (10 total points)

-Laser group: $30 \mathrm{~J}$ per leg (3 J at each site) $30 \mathrm{~s}$ at each of the 5 points on bilateral rectus femoris (10 total points)

\section{Aver Vanin et al $^{6}$}

28 male soccer athletes

Eccentric exercise protocol

$810 \mathrm{~nm}$

-Group A: $60 \mathrm{~J}$ of total irradiated energy (10 J in each site) with $10 \mathrm{~s}$ of irradiation at each site ( $60 \mathrm{~s}$ of total irradiation time) -Group B: $180 \mathrm{~J}$ of total irradiated energy ( $30 \mathrm{~J}$ in each site) with $30 \mathrm{~s}$ of irradiation at each site (180 s of total irradiation time)

-Group C: $300 \mathrm{~J}$ of total irradiated energy (50 J in each site) with $50 \mathrm{~s}$ of irradiation at each site $(300 \mathrm{~s}$ of total irradiation time)

-Group D: $0 \mathrm{~J}$ of total irradiated energy ( $0 \mathrm{~J}$ in each site) with $20 \mathrm{~s}$ of sham irradiation at each site (120 s of total time), but without effective irradiation

Treatment area

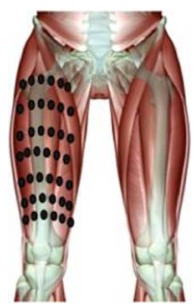

When LLLT was performed

Preperformance or postperformance LLLT

CK results

-Post-LLLT: Significantly decreased CK levels $(P<.01)$ when compared with placebo group at 10- and 15-min blood draw on both days 1 and 8

-Pre-LLLT: Significantly decreased CK levels $(P<.05)$ when compared with placebo at 10- and 15-min intervals on day 1 and 8

$\mathrm{BL}$ results

Conclusion

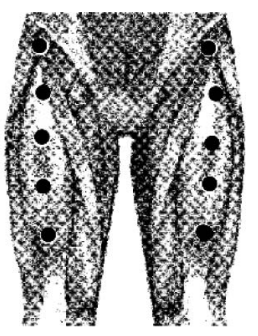

Preperformance LLLT

Not applicable

Laser group: Pre-LLLT significantly decreased BL levels $(P<.01) 15 \mathrm{~min}$ compared with placebo group.

Preexercise LLLT performed reduced BL vs placebo after $15 \mathrm{~min}$ of completing the exercise program. postexercise was completed when

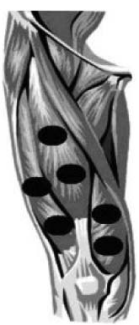

Preperformance LLLT

-Group A: 10 J LLLT dose significantly decreased $(P<.05)$ CK serum levels compared with placebo in 24-, 48-, 72-, -and 96-h time intervals.

-Group B: 30 J LLLT did not significantly decrease $(P>.05)$ CK with LLLT at $24,48,72$, and $96 \mathrm{~h}$ postexercise. -Group C: 50 J LLLT dose significantly decreased CK $(P<.05)$ at intervals of 24 , 48,72 , and $96 \mathrm{~h}$ after performance.

Not applicable

Preexercise LLLT reduced CK levels when evaluated postexercise. However, differences were not noted until $24 \mathrm{~h}$ later on 10 and $50 \mathrm{~J}$.
-Post-LLLT: Significantly decreased BL levels $(P<.01)$ when compared with placebo at 10- and 15-min intervals when compared with placebo group on days 1 and 8

-Pre-LLLT: Decreased BL levels at 10- and 15-min intervals on day 1 and 15-min interval on day 8 . Results were not clinically significant on either day.

Both pre-LLLT and post-LLLT significantly reduced $\mathrm{BL}$ and $\mathrm{CK}$ vs placebo group. The greatest differences were noted when postexercise LLLT was performed at 10- and 15-min intervals.

Abbreviations: 1-RM, 1-repetition maximum; BL, blood lactate; CK, creatine kinase; LLLT, low-level laser therapy. 


\section{References}

1. Barbosa D, Rodrigo C, Morandi RF, et al. Creatine kinase kinetics in professional soccer players during a competitive season. Rev Bras de Cineantropometria Desempenho Humano. 2011;13(3):189-194. doi:10.5007/1980-0037.2011v13n3p189

2. Bangsbo J, Iaia FM, Krustrup P. Metabolic response and fatigue in soccer. Int J Sports Physiol Perform. 2007;2(2):111-127. PubMed ID: 19124899

3. Baird M, Graham G, Baker J, Bickerstaff G. Creatine-kinase- and exercise-related muscle damage implications for muscle performance and recovery. J Nutr Metab. 2012;2012:960363. PubMed ID: 22288008 doi:10.1155/2012/960363

4. Dos Reis F, Da Silva A, Lararaia E, et al. Effects of pre- or postexercise low-level laser therapy $(830 \mathrm{~nm})$ on skeletal muscle fatigue and biochemical markers of recovery in humans: double-blind placebo-controlled trial. Photomed Laser Surg. 2014;32(2):106-112. PubMed ID: 24456143 doi:10.1089/pho.2013.3617

5. Leal-Junior E, Lopes-Martins R, Baroni B, et al. Effect of $830 \mathrm{~nm}$ low-level laser therapy applied before high-intensity exercises on skeletal muscle recovery in athletes. Lasers Med Sci. 2009;24:857. PubMed ID: 19057981 doi:10.1007/s10103-008-0633-4
6. Aver Vanin A, De Marchi T, Tomazoni S, et al. Pre-exercise infrared low-level laser therapy $(810 \mathrm{~nm})$ in skeletal muscle performance and postexercise recovery in humans, what is the optimal dose? A randomized, double-blind, placebo-controlled clinical trial. Photomed Laser Surg. 2016;34(10):473-482. PubMed ID: 27575834 doi:10.1089/pho.2015.3992

7. Stuart F. Human Physiology. 13th ed. New York, NY. McGraw Hill; 2011.

8. Powers S, Howley E. Exercise Physiology: Theory and Application to Fitness and Performance. 8th ed. New York, NY: McGraw Hill; 2012.

9. Leal-Junior E, Aver Vanin A, Miranda E, de Carvalho P, Corso S, Bjordal J. Effect of phototherapy (low-level laser therapy and lightemitting diode therapy) on exercise performance and markers of exercise recovery: a systematic review with meta-analysis. Lasers Med Sci. 2015;30(2):925-939. doi:10.1007/s10103-013-1465-4

10. De Marchi T, Schmitt VM, da Sliva Fabro CD, et al. Phototherapy for improvement of performance and exercise recovery: comparison of 3 commercially available devices. J Athl Train. 2017;52(5):429-438. PubMed ID: 28319422

11. Starkey C. Therapeutic Modalities. 4th ed. Philadelphia, PA: F.A. Davis Company; 2013. 